\title{
Off-label use: formulaire neutre de de- mande de prise en charge des coûts
}

Thomas Binder ${ }^{a}, M^{2}$ arkus Gnägi ${ }^{b}$, Herbert Plagge ${ }^{c}$, Heiner Sandmeier $^{d}$, Ursula Schafrothe ${ }^{\text {, Andreas Schiesser }}{ }^{f}$, Jürg Zollikofere

a Vips; ${ }^{\text {b }}$ santésuisse; ${ }^{\text {C GSASA; }}$ Interpharma; e SSMC; ${ }^{\mathrm{f}}$ Curafutura

\section{Off-label use}

L'off-label use (olu) de médicaments joue un rôle important en oncologie, notamment, mais aussi dans d'autres disciplines. D'une manière générale, tout ce qui ne figure pas dans le texte complet (éventuelles limitations comprises) de la liste des spécialités (LS) représente un olu, qui ne peut être pris en charge par l'assurance de base que si les conditions correspondantes de l'article 71 a à d de l'OAMal sont remplies. Lors de l'évaluation, cet article prévoit la consultation obligatoire du médecin-conseil (MC) par l'assureur. Celle-ci revêt la forme d'une évaluation au cas par cas, qui ne peut toutefois être couronnée de succès que si la demande de prise en charge des coûts est complète. Si tel est le cas, l'assureur-maladie doit rendre sa décision sous deux semaines. La Société Suisse des médecins-

\section{Un olu doit préalablement être approuvé par l'assureur.}

conseils et médecins d'assurances (SSMC) et la Conférence des sociétés cantonales de médecine (CCM) ont élaboré des règles afin d'améliorer la communication entre le demandeur et le service prestations de l'assurance. La SSMC a en outre mis au point un formulaire de demande de prise en charge des coûts ${ }^{1}$. Celui-ci reprend tous les points essentiels (tels que renseignements sur les thérapies antérieures, état clinique, pronostic, thérapie demandée et informations sur les publications relatives aux thérapies demandées). Il est très important de préciser lors de chaque demande quelles alternatives ont déjà été utilisées, s'il reste des options thérapeutiques sur la LS et pourquoi elles ne sont pas acceptables. Bien entendu, des discussions doivent également être possibles: le médecin requérant comme le MC doivent être joignables par téléphone si nécessaire.

\section{Charge administrative inutile et entrave à la liberté du médecin?}

S'il est indéniable que l'autorisation par Swissmedic et l'admission sur la LS restreignent la liberté de prescription, puisque les médicaments doivent remplir certaines conditions pour franchir ces deux obstacles, l'art 71 a à d de l'OAMal permet dans une certaine mesure l'utilisation légale d'une pharmacothérapie qui ne relève pas de la LS en tant que prestation obligatoire ou même qui n'a pas encore été autorisée en Suisse par Swissmedic.

\section{Les formulaires peuvent parfois aussi simplifier la tâche}

Un olu doit préalablement être approuvé par l'assureur. Le médecin prescripteur doit donc rédiger une demande qui doit impérativement être examinée par le MC et transmise avec une recommandation d'acceptation (ou de rejet) au service prestations de l'assureur. Afin que le MC puisse procéder à l'évaluation du bénéfice, il doit disposer de renseignements exhaustifs. Le formulaire de demande de prise en charge des coûts indique quels renseignements sont obligatoires et quels autres sont souhaités. Il est conçu pour attirer l'attention du médecin qui le complète sur les éléments manquants.

\section{Condition sine qua non?}

L'utilisation du formulaire n'est pas une condition sine qua non. Il n'existe pas de directive précisant qu'il est obligatoire de le remplir. Il inclut toutefois une option de classification qui permet de remplir la demande en un minimum de temps. Cela permet de s'épargner mutuellement du travail et de prévenir des malentendus. 